\title{
RESEARCH
}

Open Access

\section{Measuring change in trials of physical activity interventions: a comparison of self- report questionnaire and accelerometry within the PACE-UP trial}

Elizabeth S. Limb', Shaleen Ahmad ${ }^{1}$, Derek G. Cook ${ }^{1 *}$, Sally M. Kerry², Ulf Ekelund ${ }^{3}$, Peter H. Whincup ${ }^{1}$, Christina R. Victor ${ }^{4}$, Steve lliffe ${ }^{5}$, Michael Ussher ${ }^{1}$, Julia Fox-Rushby ${ }^{6}$, Cheryl Furness', Judith Ibison', Stephen DeWilde ${ }^{1}$ and Tess Harris ${ }^{1}$

\begin{abstract}
Background: Few trials have compared estimates of change in physical activity (PA) levels using self-reported and objective PA measures when evaluating trial outcomes. The PACE-UP trial offered the opportunity to assess this, using the self-administered International Physical Activity Questionnaire (IPAQ) and waist-worn accelerometry.

Methods: The PACE-UP trial $(N=1023)$ compared usual care $(n=338)$ with two pedometer-based walking interventions, by post $(n=339)$ or with nurse support $(n=346)$. Participants wore an accelerometer at baseline and 12 months and completed IPAQ for the same 7-day periods. Main outcomes were weekly minutes, all in $\geq 10 \mathrm{~min}$ bouts as per UK PA guidelines of: i) accelerometer moderate-to-vigorous PA (Acc-MVPA) ii) IPAQ moderate+vigorous PA (IPAQ-MVPA) and iii) IPAQ walking (IPAQ-Walk). For each outcome, 12 month values were regressed on baseline to estimate change.
\end{abstract}

Results: Analyses were restricted to 655 (64\%) participants who provided data on all outcomes at baseline and 12 months. Both intervention groups significantly increased their accelerometry MVPA minutes/week compared with control: postal group 42 (95\% Cl 22, 61), nurse group 43 (95\% Cl 24, 63). IPAQ-Walk minutes/week also increased: postal 57 (95\% Cl 2, 112), nurse $43(95 \% \mathrm{Cl}-11,97)$ but IPAQ-MVPA minutes/week showed non-significant decreases: postal $-11(95 \% \mathrm{Cl}-65,42)$, nurse $-34(95 \% \mathrm{Cl}-87,19)$.

Conclusions: Our results demonstrate the necessity of using a questionnaire focussing on the activities being altered, as with IPAQ-Walk questions. Even then, the change in PA was estimated with far less precision than with accelerometry. Accelerometry is preferred to self-report measurement, minimising bias and improving precision when assessing effects of a walking intervention.

Trial registration: ISRCTN, ISRCTN98538934. Registered 2 March 2012.

Keywords: Walking, Intervention, Primary care, MVPA, Accelerometry, IPAQ, GPPAQ

\footnotetext{
* Correspondence: d.cook@sgul.ac.uk

'Population Health Research Institute, St George's University of London,

Cranmer Terrace, Tooting, London SW17 ORE, UK

Full list of author information is available at the end of the article
}

(c) The Author(s). 2019 Open Access This article is distributed under the terms of the Creative Commons Attribution 4.0 International License (http://creativecommons.org/licenses/by/4.0/), which permits unrestricted use, distribution, and reproduction in any medium, provided you give appropriate credit to the original author(s) and the source, provide a link to the Creative Commons license, and indicate if changes were made. The Creative Commons Public Domain Dedication waiver (http://creativecommons.org/publicdomain/zero/1.0/) applies to the data made available in this article, unless otherwise stated. 


\section{Background}

Adults who participate in regular physical activity (PA) and remain fit and active into later life have fewer chronic health conditions, and are better able to maintain a healthy weight [1]. WHO, UK and US aerobic PA guidelines for adults recommend at least 150 min weekly of moderate-to-vigorous-physical-activity (MVPA) in bouts of at least $10 \mathrm{~min}$, or $75 \mathrm{~min}$ of vigorous $\mathrm{PA}$, or a combination. Brisk walking ( 3 miles/hr. or $5 \mathrm{~km} / \mathrm{hr}$ ) counts as MVPA [2] and for most people approximates to 1000 steps in $10 \mathrm{~min}$ [3].

Self-report questionnaires are a quick, easy way to assess PA. Population surveys such as the Health Survey for England (HSE) [4] and Sport England's "Active Lives Survey" [5] use self-completed questionnaires and report estimates that around $60 \%$ of participants aged $16+$ meet PA guidelines. However, individuals often over-estimate their PA, particularly walking, on questionnaires compared with accelerometry measures of MVPA [6-8]. Self-report questionnaires can thus lead to inflated estimates of "active" individuals [9].

The International Physical Activity Questionnaire (IPAQ) short form [10] assesses 7-day recall of PA in $\geq 10$ min bouts based on intensity (separating vigorous, moderate and walking activity) and duration (days per week and minutes per day). The shorter General Practice Physical Activity Questionnaire (GPPAQ) [11] does not provide a continuous measure of $\mathrm{PA}$, but categorises individuals as active or not. GPPAQ is used in the UK National Health Service (NHS) primary care cardiovascular health checks [12]. Individuals classified as less than "active" are assumed not to be meeting PA guidelines and are offered advice. In contrast, accelerometry is an objective PA measure, providing information on step-counts and time spent in different PA intensities and is increasingly being used in cross-sectional studies to study PA $[13,14]$. Although accelerometers e.g. Actigraph are not a gold standard for measuring PA, they have been shown to correlate well with doubly labelled water to measure activity energy expenditure [15]. For the Actigraph GT3X accelerometer, standard cut-points for accelerometer counts per minute (CPM) for different PA intensity categories have also been defined, thus leading to assessment of time spent in different PA intensities: light 101-1951 CPM; moderate 1952-5724 CPM; vigorous $\geq 5725$ CPM [16].

Longitudinal studies and trials which examine PA changes over time need valid, reliable PA assessment methods. Both IPAQ-Short and accelerometry have been used separately to measure PA change over time, [1720] but only a few small studies have used both and compared change in minutes of PA [21, 22]. Other studies have compared self-report PA minutes with either pedometer steps [23, 24] or accelerometry counts [25] which are not directly comparable. The PACE-UP trial offers the opportunity to directly compare change in PA minutes from accelerometry and IPAQ within a large trial dataset. This study had the following objectives: to compare the trial treatment effects at 12 months (difference between intervention and control groups in the change in PA) using (i) accelerometry minutes of MVPA and IPAQ minutes of moderate+vigorous activity and walking; (ii) the percentage of "active" individuals classified by accelerometry, IPAQ and GPPAQ.

\section{Methods}

\section{Background to the PACE-UP study}

The PACE-UP study is a three-arm parallel groups randomised controlled trial comparing a 3-month pedometer-based walking intervention, delivered by post or with nurse support, to usual care [26]. Ethical approval was given by the London Research Ethics Committee (Hampstead) (12 L/LO/0219), trial registration ISRCTN 98538934. Adults aged 45-75 years from seven South-West London (UK) General Practices (family practices) who self-reported as inactive were invited to take part. Following a baseline assessment to assess eligibility, 1023 participants gave informed written consent and were randomised into one of three groups: the Control group $(n=338)$ received usual care; the Postal group $(n=339)$ received a pedometer, a 12 -week personalised walking plan including behaviour change techniques (e.g. goal setting, self-monitoring) designed to increase their walking and a step-count diary through the post; the Nurse group $(n=346)$ received these and were additionally offered three individual practice nurse PA consultations. Randomisation was carried out at household level allowing couples to take part together. The main trial outcomes were changes in accelerometry measured average daily step-count and total weekly time in MVPA in $\geq 10 \mathrm{~min}$ bouts between baseline and 12 months. 956/ 1023 (93\%) provided at least 1 day of accelerometry data at 12 months, $>90 \%$ provided at least 5 days wear. The postal and nurse groups both significantly increased their objective PA levels (step count and time in MVPA) compared with the control group, with no difference between intervention groups at 12 months [27].

Participants wore a sealed accelerometer (GT3X, Actigraph LLC) over their hip for 7 consecutive days at baseline, prior to randomization, and 12 months post-randomization. They also completed the IPAQ Short form [10] and GPPAQ [11], both designed for self-completion, for the same 7-day periods as they wore the accelerometer. Actilife software (v 6.6.0) was used to extract and reduce the Actigraph data, ignoring runs of $\geq 60 \mathrm{~min}$ of zero counts [26], to provide daily steps counts and time spent in $\geq 10 \mathrm{~min}$ bouts of MVPA $(\geq 1952$ counts per minute, equivalent to $\geq 3$ Metabolic 
Equivalents (METs)) [16]. When assessing $\geq 10 \mathrm{~min}$ bout, the default "drop time" of 2 min was used, which allows for a 2 min interruption in bout activity. At baseline, all participants provided $\geq 5$ days of $\geq 540$ min accelerometer wear-time. To limit attrition bias, those providing $\geq 1$ day of $\geq 540 \mathrm{~min}$ accelerometer wear time at 12 months were included in analyses. IPAQ questions focus on time spent being physically active in the previous 7 days in at least 10 min bouts, including PA at work, home, travelling and leisure. For each of vigorous and moderate PA and walking, there are questions on the number of days and the duration on each of these days. GPPAQ questions ask about PA at work and the type and weekly duration of leisure PA (physical exercise/sport, cycling, walking, housework/childcare and gardening/DIY). Duration categories are None, $<1 \mathrm{~h}, 1-3 \mathrm{~h}, \geq 3 \mathrm{~h}$.

\section{Study outcomes}

\section{Accelerometry}

The main accelerometry outcome was total weekly minutes of MVPA in $\geq 10$ min bouts; a secondary outcome was total weekly minutes of MVPA, including MVPA in $<10$ min bouts. Binary variables were generated for each MVPA outcome to indicate 150 min of activity.

\section{IPAQ}

Total weekly minutes spent in each of vigorous PA, moderate PA and walking were calculated, capped at a maximum of $3 \mathrm{~h} /$ day or $21 \mathrm{~h} /$ week, as recommended by the IPAQ coding guidelines [28]. Two self-report PA measures were derived: total weekly minutes of vigorous + moderate PA in bouts of $\geq 10 \mathrm{~min}$, excluding walking (IPAQ-MVPA) and total weekly minutes of walking in bouts of $\geq 10$ min (IPAQ-Walk). We also report an additional outcome, IPAQ-Total (IPAQ-MVPA + IPAQ-Walk), conceptually the same construct as accelerometry MVPA in $\geq 10$ min bouts. Binary variables were generated for each of these to indicate $150 \mathrm{~min}$ or more per week of activity.

\section{GPPAQ}

The GPPAQ Physical Activity Index is a 4-level index ranging from "Inactive" through to "Active". "Active" individuals are achieving $\geq 3 \mathrm{~h}(180 \mathrm{~min})$ of MVPA per week including work PA and leisure PA from physical exercise and cycling, but not including PA from walking, housework/childcare or gardening. We defined a binary outcome, GPPAQ, to identify those individuals classified as "Active" by the GPPAQ score. However, adults who are retired or not working and who do no sport or cycling can never be classified as active, although they may achieve MVPA guidelines through walking. Thus, a modified index, GPPAQ-Walk, was also derived, where those who reported walking briskly for at least $3 \mathrm{~h}$ per week were classified as "active". Previous analysis of GPPAQ showed this modified index had improved sensitivity at identifying active individuals compared with accelerometry data, but lower specificity in adults aged $60-75$ years [29].

\section{Statistical analyses}

Analyses were carried out using Stata 14 [30]. Multi-level regression models estimated treatment effects for accelerometer, IPAQ and GPPAQ outcomes. The 12-month outcome was regressed on baseline value, treatment group, age, gender, practice and month of baseline accelerometry as fixed effects and household as a random effect in the multi-level model. (i) Linear regression was used for weekly minutes of accelerometer MVPA, IPAQ-MVPA, IPAQ-Walk and IPAQ-Total; (ii) logistic regression was used for the binary variables "active" from accelerometry, IPAQ and GPPAQ. The distributions of change in PA for the four continuous outcomes were reasonably normally distributed, as were the distributions of residuals from the models, allowing this method of analysis. Analyses were restricted to those with complete data for all outcomes being compared: 833 at baseline and 655 for the longitudinal regression models. This ensured direct comparisons of the same group of participants for each outcome. Sensitivity analyses used $\geq 180 \mathrm{~min}$ of accelerometer MVPA and IPAQ outcomes, as the GPPAQ outcome is based on $\geq 180$ min per week.

\section{Results}

At baseline, accelerometry data were available on all participants and 989 (97\%) returned IPAQ and GPPAQ questionnaires. At 12 months, 956 (93\%) participants provided at least 1 day of accelerometry and 942 (92\%) returned IPAQ and GPPAQ questionnaires. However, incomplete answers on IPAQ and GPPAQ questions reduced the sample size to 833 at baseline and to 655 for analyses of changes between baseline and 12 months. Study groups were balanced at baseline for the 833 with complete data with respect to age, gender, ethnicity and different health measures (Table 1). One third of participants were male and two thirds were overweight or obese (Body Mass Index $\geq 25 \mathrm{~kg} / \mathrm{m}^{2}$ ). Mean weekly minutes of accelerometer-MVPA were 317 (sd 151) for total MVPA and 98 (sd 103) for MVPA in $\geq 10 \mathrm{~min}$ bouts. Self-reported mean weekly minutes were 174 (sd 279) for IPAQ-MVPA, 315 (sd 310) for IPAQ-Walk, similar to total accelerometry MVPA and 489 (sd 453) for IPAQ-Total. Accelerometry data classified 23\% of participants at baseline as "Active" i.e. achieving $\geq 150$ min of MVPA per week in $\geq 10 \mathrm{~min}$ bouts (Table 1). In contrast, 35, 66 and $84 \%$ of participants self-reported $\geq 150$ min per week of IPAQ-MVPA, IPAQ-Walk and 
Table 1 Demographic, health, physical characteristics and physical activity at baseline

\begin{tabular}{|c|c|c|c|c|}
\hline & $\begin{array}{l}\text { All groups } \\
(N=833)\end{array}$ & $\begin{array}{l}\text { Control } \\
(N=279)\end{array}$ & $\begin{array}{l}\text { Postal } \\
(N=270)\end{array}$ & $\begin{array}{l}\text { Nurse } \\
(N=284)\end{array}$ \\
\hline & n (\%) & n (\%) & n (\%) & n (\%) \\
\hline \multicolumn{5}{|l|}{ Age at randomisation } \\
\hline $45-54$ years & $280(34 \%)$ & 87 (31\%) & $94(35 \%)$ & $99(35 \%)$ \\
\hline $55-64$ years & $315(38 \%)$ & $111(40 \%)$ & $98(36 \%)$ & $106(37 \%)$ \\
\hline $65-75$ years & $238(29 \%)$ & $81(29 \%)$ & 78 (29\%) & 79 (28\%) \\
\hline Sex: Male & $304(36 \%)$ & $98(35 \%)$ & $104(39 \%)$ & $102(36 \%)$ \\
\hline \multicolumn{5}{|l|}{ Ethnicity } \\
\hline White & $654(81 \%)$ & $212(79 \%)$ & $222(85 \%)$ & $220(80 \%)$ \\
\hline Black / African / Caribbean / Black British & 77 (10\%) & $25(9 \%)$ & $21(8 \%)$ & $31(11 \%)$ \\
\hline Asian / Asian British & $54(7 \%)$ & $21(8 \%)$ & $14(5 \%)$ & $19(7 \%)$ \\
\hline Other, incl mixed & $19(2 \%)$ & $10(4 \%)$ & $4(2 \%)$ & $5(2 \%)$ \\
\hline General health: Very good or good & $679(83 \%)$ & $223(81 \%)$ & $230(88 \%)$ & $226(82 \%)$ \\
\hline \multicolumn{5}{|l|}{ Chronic diseases } \\
\hline None & $321(39 \%)$ & $109(39 \%)$ & $112(42 \%)$ & $100(36 \%)$ \\
\hline $1-2$ & $436(53 \%)$ & $153(55 \%)$ & $133(50 \%)$ & $150(54 \%)$ \\
\hline$\geq 3$ & $61(7 \%)$ & $14(5 \%)$ & $20(8 \%)$ & $27(10 \%)$ \\
\hline Self-reported pain: Yes & $566(69 \%)$ & $185(67 \%)$ & $191(72 \%)$ & $190(69 \%)$ \\
\hline Limiting long-standing illness & $174(21 \%)$ & $60(22 \%)$ & $55(21 \%)$ & $59(21 \%)$ \\
\hline \multicolumn{5}{|l|}{ Townsend Disability score } \\
\hline None (0) & $491(60 \%)$ & $159(58 \%)$ & $158(59 \%)$ & $174(62 \%)$ \\
\hline Slight or some disability (1-6) & $305(37 \%)$ & $103(37 \%)$ & $104(39 \%)$ & $98(35 \%)$ \\
\hline Appreciable or severe disability $(7-18)$ & $24(3 \%)$ & $13(5 \%)$ & $4(2 \%)$ & $7(3 \%)$ \\
\hline \multicolumn{5}{|l|}{ Physical characteristics } \\
\hline \multirow[t]{2}{*}{ Overweight/obese: $\mathrm{BMI} \geq 25 \mathrm{~kg} / \mathrm{m}^{2}$} & $544(65 \%)$ & $184(66 \%)$ & $173(64 \%)$ & $187(66 \%)$ \\
\hline & Mean (sd) & Mean (sd) & Mean (sd) & Mean (sd) \\
\hline Fat mass (kg) & $26(11)$ & $26(10)$ & $26(11)$ & $26(11)$ \\
\hline \multicolumn{5}{|l|}{ Accelerometry data } \\
\hline Adjusted baseline step count per day & $7550(2670)$ & $7528(2685)$ & $7480(2583)$ & $7638(2744)$ \\
\hline Total weekly mins MVPA in $\geq 10$ min bouts & $98(103)$ & $91(100)$ & $97(94)$ & $106(113)$ \\
\hline Total weekly mins MVPA & $317(151)$ & $316(152)$ & $311(145)$ & $322(154)$ \\
\hline Daily wear time (mins) & $792(79)$ & $791(73)$ & $789(79)$ & $796(84)$ \\
\hline \multicolumn{5}{|l|}{ International Physical Activity Questionnaire (IPAQ) } \\
\hline IPAQ-MVPA: Weekly mins of moderate PA + vigorous PA in $\geq 10$ min bouts & $174(279)$ & $194(310)$ & $159(266)$ & $167(259)$ \\
\hline IPAQ-Walk: Weekly mins of walking in $\geq 10$ min bouts & $315(310)$ & $323(327)$ & $316(326)$ & $307(275)$ \\
\hline \multirow[t]{2}{*}{ IPAQ-Total: Weekly mins of moderate PA + vigorous PA + walking in $\geq 10$ min bouts } & $489(453)$ & $518(501)$ & $475(457)$ & $474(395)$ \\
\hline & n (\%) & n (\%) & n (\%) & n (\%) \\
\hline \multicolumn{5}{|l|}{ Proportions of "active" individuals ${ }^{a}$} \\
\hline \multicolumn{5}{|l|}{ Accelerometry } \\
\hline 150 weekly mins MVPA in $\geq 10$ min bouts & $190(23 \%)$ & $57(21 \%)$ & $58(22 \%)$ & 75 (27\%) \\
\hline \multicolumn{5}{|l|}{ International Physical Activity Questionnaire (IPAQ) } \\
\hline 150 weekly mins of IPAQ-MVPA & $286(35 \%)$ & $99(36 \%)$ & $86(32 \%)$ & $101(36 \%)$ \\
\hline 150 weekly mins of IPAQ-Walk & $540(66 \%)$ & $176(64 \%)$ & $173(65 \%)$ & $191(68 \%)$ \\
\hline 150 weekly mins of IPAQ-Total & $690(84 \%)$ & $227(82 \%)$ & $226(85 \%)$ & $237(84 \%)$ \\
\hline
\end{tabular}


Table 1 Demographic, health, physical characteristics and physical activity at baseline (Continued)

\begin{tabular}{|c|c|c|c|c|}
\hline & $\begin{array}{l}\text { All groups } \\
(N=833)\end{array}$ & $\begin{array}{l}\text { Control } \\
(N=279)\end{array}$ & $\begin{array}{l}\text { Postal } \\
(N=270)\end{array}$ & $\begin{array}{l}\text { Nurse } \\
(N=284)\end{array}$ \\
\hline & n (\%) & n (\%) & n (\%) & n (\%) \\
\hline \multicolumn{5}{|l|}{ General Practice Physical Activity Questionnaire (GPPAQ) } \\
\hline GPPAQ: "Active" $\geq 180$ mins PA per week & $101(12 \%)$ & $38(14 \%)$ & $33(12 \%)$ & $30(11 \%)$ \\
\hline GPPAQ-Walk: "Active" $\geq 180$ mins PA per week including walking at brisk/fast pace & $229(28 \%)$ & $82(30 \%)$ & $71(27 \%)$ & $76(27 \%)$ \\
\hline
\end{tabular}

aproportions of "active" individuals are based on 276, 265 and 281 participants in Control, Postal and Nurse groups respectively

IPAQ-Total respectively. GPPAQ classified $12 \%$ of participants as active which increased to $28 \%$ when walking was included.

\section{i) Comparison of estimated treatment effects using minutes of physical activity}

Both intervention groups showed statistically significant increases in accelerometer-MVPA, both in bouts and total, compared with controls. Increases in accelerometer-MVPA bouts: postal group $42 \mathrm{~min} /$ week (95\% CI 22 to 61), nurse group 43 (95\% CI 24 to 63 ) (Table 2 and Fig. 1a); increases for total accelerometry MVPA were almost identical to accelerometer-MVPA in bouts but with wider confidence intervals (Table 2 and Fig. 1). Repeating the analysis using the IPAQ outcomes, IPAQ-Walk showed positive increases, similar in magnitude to accelerometer-MVPA in the nurse group, but with wider confidence intervals indicating less precision: postal group $57 \mathrm{~min}$ (95\% CI 2 to 112), nurse group 43 (95\% CI -11 to 97). IPAQ-MVPA showed non-significant decreases and IPAQ-Total showed non-significant increases. The distribution of residuals from the regression models were normally distributed for MVPA in bouts [27] and IPAQ outcomes (data not shown).

\section{ii) Comparison of estimated treatment effects using the binary variable "active"}

Similar patterns were found for the binary variable "active" for the different outcomes. Odds ratios (ORs) for being "active" at 12 months (achieving $\geq 150$ weekly minutes of MVPA in $\geq 10$ min bouts) conditional on baseline "active" status were statistically significant for accelerometry-MVPA: postal group 3.7 (95\% CI 1.8 to 7.5) and nurse group 2.9 (95\% CI 1.5 to 5.7 ) (Table 3). IPAQ-Walk showed statistically significant OR for the postal group, 2.1 (95\% CI 1.2 to 4.0 ) and borderline for the nurse group, 1.7 (95\% CI 1.0 to 3.0). Results were inconclusive for IPAQ-MVPA and IPAQ-Total had increased ORs for both intervention groups, but only statistically significant for the nurse group. ORs for the two GPPAQ outcomes were close to 1.0 suggesting that GPPAQ was unable to identify changes in the proportion classified as "active" (Table 3). Sensitivity analyses using $\geq 180 \mathrm{~min}$ of the accelerometer and IPAQ outcomes gave similar results.

\section{Discussion}

The PACE-UP study was a walking intervention designed to increase individuals' PA through a 3-month programme, in particular MVPA in $\geq 10 \mathrm{~min}$ bouts in line with current UK, WHO and US PA guidelines [31-33]. We found statistically significant increases between baseline and 12 months in accelerometer measured MVPA in $\geq 10$ min bouts for both intervention groups compared with control. IPAQ-Walk showed a significant increase in the postal group and a non-significant increase in the nurse group compared with control, but with less precision than with accelerometry. IPAQ-MVPA showed non-significant decreases and IPAQ-Total non-significant increases in intervention groups compared with controls. When considering the proportion of "active" individuals, only accelerometry showed statistically significant increases for both intervention groups versus controls. IPAQ-Walk and IPAQ-Total showed statistically significant increases for one intervention group compared with controls (postal for IPAQ-Walk and nurse for IPAQ-Total), but borderline effects for the other intervention group compared with controls. Neither IPAQ-MVPA nor GPPAQ identified any change in the proportions categorised as "active" in intervention versus control groups. Therefore, in terms of overall construct validity for assessing change in walking in a walking intervention study, accelerometry has the greatest validity, followed by IPAQ-Walk. The other measures have considerable disadvantages: IPAQ-MVPA and GPPAQ have very poor construct validity; IPAQ-Total is measured with substantial imprecision and is unsuitable for assessing a walking intervention as it includes IPAQ-MVPA.

Our study had several strengths. It was based on a large population-based sample of adults from seven south-west London (UK) general practices (family practices), predominantly classified as inactive at baseline. Accelerometry is an objective PA measure and measures walking accurately. We used standard cut-points to define the different intensities of accelerometry activity and were thus able to identify those bouts of walking which can be classified as MVPA. The main PACE-UP analysis 


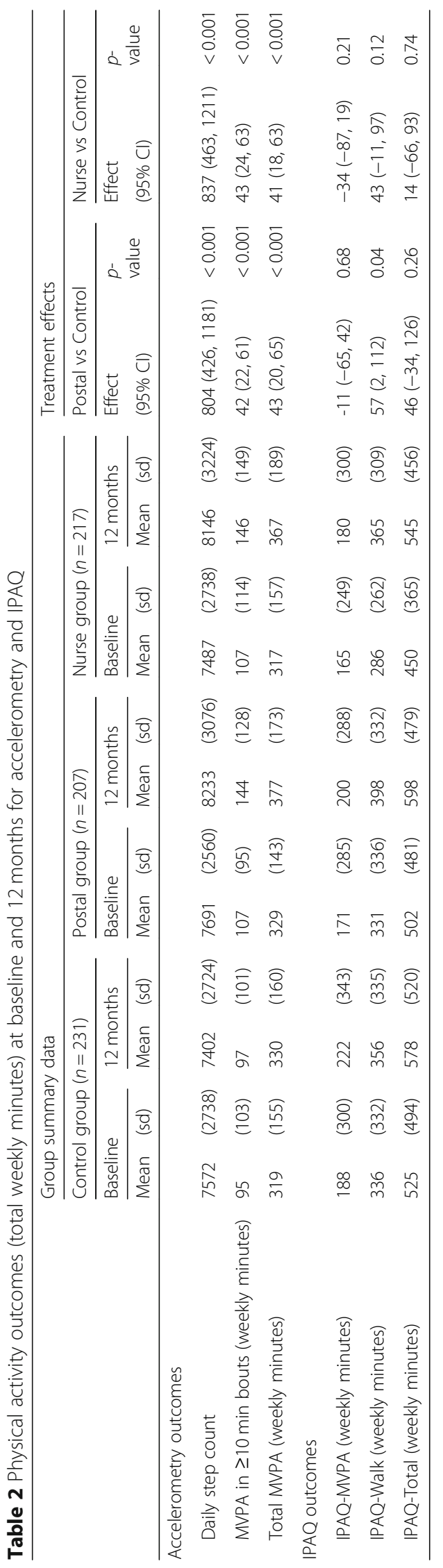




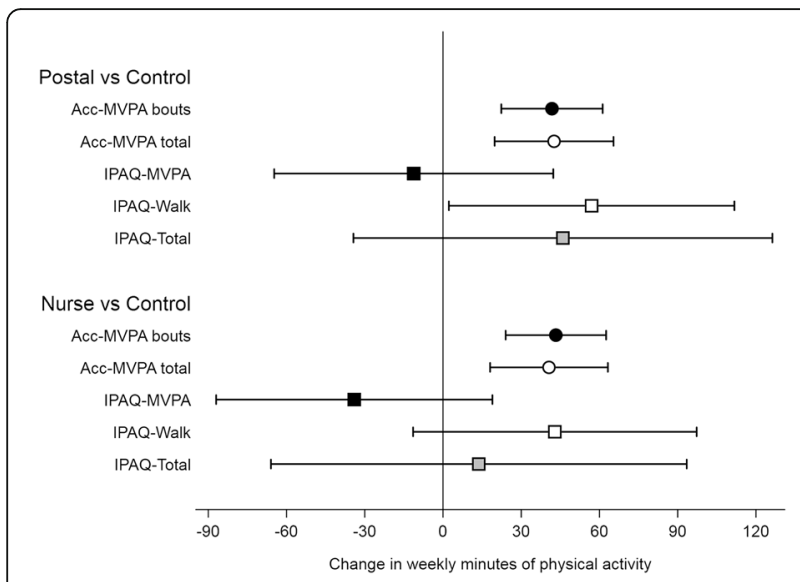

Fig. 1 Treatment effects and 95\% confidence intervals for change in minutes of physical activity measured by accelerometry, IPAQ-MVPA, IPAQ-Walk and IPAQ-Total

[27] showed that the increase in weekly steps in intervention groups relative to control group was equivalent to the increase in weekly minutes of MVPA and this was all in $\geq 10$ min bouts, thus demonstrating the effectiveness of the PACE-UP walking intervention. The two self-completed questionnaires, IPAQ and GPPAQ, are standard questionnaires used to assess PA, and were completed for the same 7 days as for accelerometry, thus providing directly comparable estimates of effect. The study achieved 93\% accelerometry follow-up at 12 months, $>90 \%$ of these with $\geq 5$ days wear-time. Total weekly minutes of MVPA and total weekly minutes of walking (not including MVPA) were easy to extract from IPAQ and provided a direct comparison with minutes of accelerometer-MVPA. The increases in IPAQ-Walk minutes are similar to those for accelerometer-MVPA suggesting that IPAQ can identify changes in walking minutes, although the wider confidence intervals show the loss of precision from using IPAQ. At baseline, average IPAQ-Walk minutes were similar to average total accelerometer-MVPA minutes rather than accelerometer-MVPA in $\geq 10 \mathrm{~min}$ bouts. This is perhaps unsurprising, as the IPAQ walking questions ask for number of days walking and duration on each day, and people may find it easier to report walking minutes as a rounded number e.g. 30 or $45 \mathrm{~min}$ per day and which may include relative short walks of $<10 \mathrm{~min}$. GPPAQ is commonly used in UK general practice to assess an individual's PA. However, it can underestimate PA amongst those not working or those whose main PA is walking, and this study provided a further opportunity to evaluate our modified GPPAQ-Walk index [29]. We were also able to estimate how well GPPAQ could identify individuals moving from "not active" to "active" (assumed to be achieving PA guidelines). Finally, our method of analysis, regressing outcome at 12 months on baseline values focusses on individual changes in activity while allowing for regression to the mean. Cross-sectionally, the distributions of accelerometer-MVPA and IPAQ measures are highly skewed leading many to present medians and interquartile ranges of activity at different time points. However, change in activity is usually symmetric and reasonably normally distributed, which our approach exploits. We were thus able to present mean changes in activity and associated confidence intervals for both accelerometry and questionnaire measures, thus allowing for a more informative comparison.

The study also had some important limitations. All of the PA measures (accelerometry, IPAQ and GPPAQ) only measured PA levels for 7 days and it may be that participants were more likely to be active or report being active in the week that their PA was being assessed, rather than at other times. However, any such tendency would potentially affect all of the PA measures and would be true for control participants as well as for those in the intervention group. IPAQ is difficult to complete and thus unreliable if an individual's PA varies by day across the week. Although we had high return rates at baseline and 12 months for the IPAQ and GPPAQ, 97\% and 92\% respectively, each IPAQ outcome at baseline and 12 months had $20-25 \%$ missing or incomplete answers. Participants' comments on the questionnaires described their confusion over how to interpret and answer the questions and many questions were left blank. This reduced our sample size to 655 for comparisons with accelerometry although this is still large compared with other studies [21-23]. The proportions of missing data were similar across the three groups, but those with missing IPAQ data had lower mean accelerometry-MVPA at baseline and 12 months than those with complete data. The accelerometry effect sizes reported here (42-43 $\mathrm{min})$ are also larger than for the full cohort (33-35 $\mathrm{min})$ [27]. The limited options on GPPAQ for duration of PA, led to using $\geq 3 \mathrm{~h}(180 \mathrm{~min})$ for GPPAQ "active" whereas the PA guidelines are $\geq 150$ min. However, ORs from sensitivity analyses using $\geq 180$ min for accelerometry and IPAQ outcomes were similar to those using $\geq 150 \mathrm{~min}$. Although neither of our methods of measuring PA are considered a gold-standard, accelerometry has the advantage of providing an objective time-stamped record of PA that does not rely on recall. It has been validated as a measure of activity energy expenditure using doubly labelled water [15] and we used standard cut-points in counts per minute to define MVPA. [16] Our findings that accelerometer-MVPA and IPAQ-Walk provide similar estimates of change clearly support results from the PACE-UP intervention which is aimed at increasing walking, but it is unknown if these findings would be generalisable to other PA interventions. 


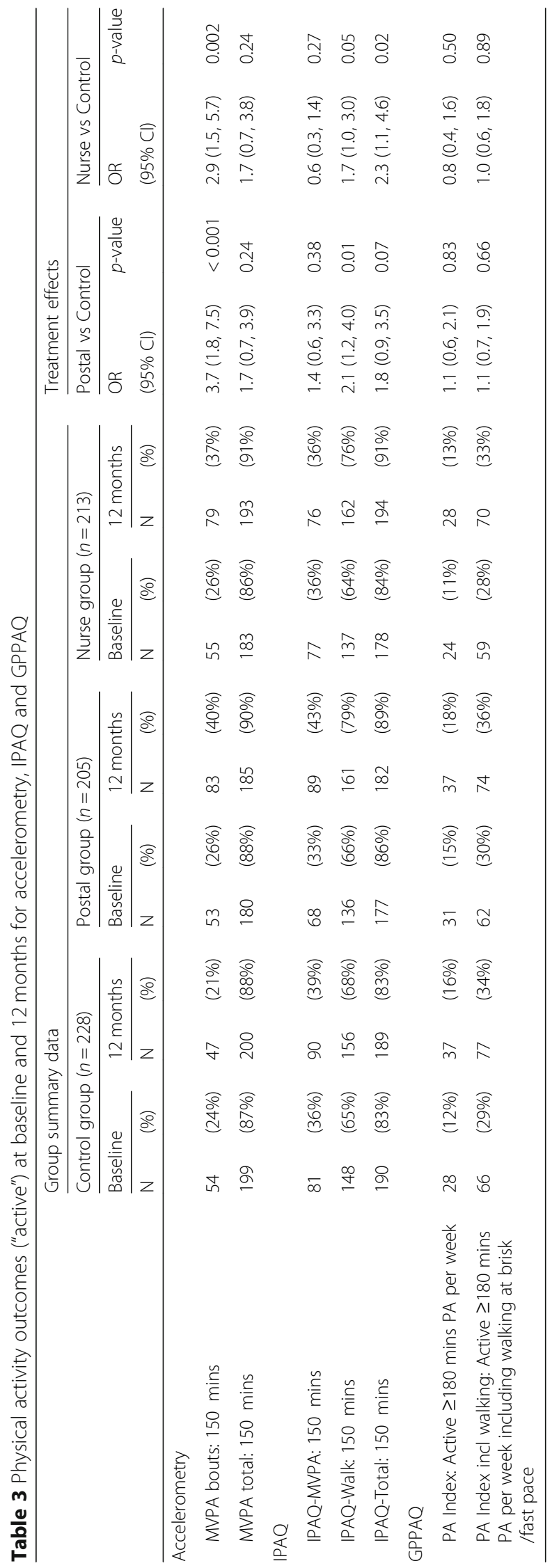


Participants in the postal and nurse intervention groups were encouraged to increase their MVPA through walking and the nurse group in particular were taught to recognise and classify different PA intensities - vigorous, moderate, light, and sedentary. Thus they may have been more likely to accurately report their PA on IPAQ at follow-up i.e. with less over-estimation of their PA levels, which could explain the non-significant decreases in the treatment groups for IPAQ-MVPA from the modelling.

\section{Comparison with other studies}

Our baseline data agree with other studies that individuals tend to over-estimate their PA on self-report questionnaires compared with objective accelerometry, both time spent being physically active [6] and proportions achieving PA guidelines [7]. Studies which have found better correspondence between IPAQ and accelerometry cross-sectionally [34] have used total accelerometer MVPA rather than MVPA in $\geq 10$ min bouts and a similar pattern is seen in our data where baseline total accelerometer-MVPA minutes are similar to IPAQ-Walk minutes. However, IPAQ questions ask about vigorous and moderate PA in $\geq 10$ min bouts and UK, WHO and US PA guidelines are based on $\geq 150 \mathrm{~min}$ of MVPA per week in $\geq 10$ min bouts. In our trial, whilst total accelerometry MVPA was much higher than accelerometry MVPA in $\geq 10$ min bouts, changes in both measures were almost identical.

To our knowledge, this is the largest population-based trial to make direct comparisons of accelerometry and self-report questionnaires to assess an individual's change in minutes of PA after an intervention. There are limitations with all five studies we identified [21-25] which have attempted to compare longitudinal changes in PA measured using IPAQ compared with objective measures. Three studies recruited less than 100 subjects [21-23]. One study was observational [21], one had no control group [22] and one was a weight loss intervention rather than PA intervention [24]. One study was comparing IPAQ with pedometer steps [23] and another with accelerometer counts [25] making direct comparison of minutes of physical activity between IPAQ and accelerometry difficult. Whilst our study compares measures using different constructs, we were able to compare time spent in MVPA and time spent walking, both in minutes per week. Three studies present distribution of PA measures at baseline and follow-up, but provided no estimate of the distribution of change [21, 24, 25]. Our findings do agree with two of the small studies. Nicaise et al. [22] followed up one group of women, but with no control group, and found median changes in IPAQ Walking minutes were similar to median changes in accelerometer MVPA minutes. Baker et al. [23] compared IPAQ PA minutes with pedometer steps, and argue that the increase in step counts in the intervention group was comparable to the increase in leisure time walking reported on IPAQ, although they report mean differences for pedometer steps and median differences for IPAQ data.

GPPAQ is used in UK primary care to help identify those not achieving PA guidelines during UK NHS Health Checks [12]. GPPAQ guidance recommends repeating it annually on those at increased cardiovascular risk [11], but our study suggests that it is poor at identifying those individuals who have increased their PA to current guideline levels. In addition, the binary nature of this outcome fails to recognise modest, but important, increases in PA made by inactive individuals. We have also confirmed our previous findings [29] that, compared with objective accelerometry, GPPAQ underestimates the proportion of "active" individuals and our modified index GPPAQ-Walk classifies slightly more as "active".

\section{Conclusions}

We have demonstrated that neither GPPAQ nor IPAQ-MVPA provide a valid estimate of change in a walking intervention trial compared with accelerometry measures. Moreover, we have shown that although IPAQ-Walk produces an estimate of change comparable with that from accelerometry MVPA in $\geq 10$ min bouts, the IPAQ-Walk estimate had considerably less precision. Missing data were also an issue with the self-report IPAQ. This has implications for future trials. Studies may need to use IPAQ to assess changes in walking if they are not able to use accelerometry. If this is the case, they should focus particularly on the walking questions and will need to be larger to be adequately powered, although they will still lack information on intensity of any changes that occur. In conclusion, accelerometry is preferred to self-report measures in assessing the effects of a walking intervention, as it avoids recall bias and improves precision.

\section{Abbreviations}

95\% Cl: 95\% Confidence Interval; GPPAQ: General Practice Physical Activity Questionnaire; IPAQ: International Physical Activity Questionnaire;

MVPA: Moderate-to-vigorous physical activity; NHS: UK National Health Service; OR: Odds ratio; PA: Physical activity; sd: Standard deviation

\section{Acknowledgements}

We would like to thank the South-West London (UK) general practices, their practice nurses who supported this study, and all the patients from these practices who participated: Upper Tooting Road Practice, Tooting; Chatfield Practice, Battersea; Wrythe Green Practice, Carshalton; Francis Grove Practice, Wimbledon; Putneymead Practice Putney; Heathfield Practice Putney; and Cricket Green Practice, Mitcham.

\section{Funding}

The research was conducted as part of the main randomised controlled trial of PACE-UP which was funded by the Health Technology Assessment Programme, National Institute of Health Research UK, (project number HTA 10/32/02, ISRCTN98538934) and has been published in full in Health 
Technology Assessment (DOI https://doi.org/10.3310/hta22370). The funders had no role in study design (beyond the commissioned call outline), data collection and analysis, decision to publish, or preparation of the manuscript.

\section{Availability of data and materials}

There are restrictions on the availability of the data for this study due to the signed consent agreements around data security, which only allow access to external researchers for research monitoring purposes. Requestors wishing to access the data for the purposes of replicating or checking analyses can apply to Research Data Management at St George's University of London researchdata@sgul.ac.uk.

\section{Disclaimer}

The views and opinions expressed therein are those of the authors and do not necessarily reflect those of the Health Technology Assessment (HTA) Programme, National Institute for Health Research (NIHR) National Health Service, or the Department of Health.

The results of the study are presented clearly, honestly and without fabrication, falsification or inappropriate data manipulation.

\section{Authors' contributions}

TH, DGC, ESL and SA conceived the idea for the paper. TH, DGC, SMK, CRV, PHW, MU, SI, UE and JFR were trial investigators and designed and were awarded funding for the trial. TH was trial Principal Investigator and had overall responsibility for the execution of the project. JI and SDW recruited general practices to the trial. CF was trial manager and oversaw data collection for the trial. ESL and DGC designed the analyses and ESL conducted the analyses for this paper. The manuscript was prepared by $\mathrm{TH}$ ESL and DGC with input from all of the other authors. All of the author team reviewed and approved the manuscript prior to submission.

\section{Ethics approval and consent to participate}

Ethics approvals were obtained from NRES Committee London - Hampstead REC reference: 12/LO/0219. Written consent was obtained from all participants.

\section{Consent for publication}

Not applicable.

\section{Competing interests}

The authors declare that they have no competing interests.

\section{Publisher's Note}

Springer Nature remains neutral with regard to jurisdictional claims in published maps and institutional affiliations.

\section{Author details}

'Population Health Research Institute, St George's University of London, Cranmer Terrace, Tooting, London SW17 ORE, UK. ${ }^{2}$ Pragmatic Clinical Trials Unit, Queen Mary's University of London, London E1 2AT, UK. ${ }^{3}$ Department of Sport Medicine, Norwegian School of Sport Sciences, PO Box 4014, 0806 Oslo, Norway. ${ }^{4}$ Gerontology and Health Services Research Unit, Brunel University, London UB8 3PH, UK. ${ }^{5}$ Research Department of Primary Care \& Population Health, University College, London NW3 2PF, UK. ${ }^{6}$ Present address: Department of Population Health Sciences, King's College London, London SE1 1UL, UK.

\section{Received: 27 March 2018 Accepted: 6 December 2018}

\section{Published online: 22 January 2019}

\section{References}

1. Lee IM, Shiroma EJ, Lobelo F, Puska P, et al. Effect of physical inactivity on major non-communicable diseases worldwide: an analysis of burden of disease and life expectancy. Lancet. 2012;380(9838):219-29.

2. Morris JN, Hardman AE. Walking to health. Sports Med. 1997;23(5):306-32.

3. Tudor-Locke C, Craig CL, Brown WJ, Clemes SA, et al. How many steps/day are enough? For adults. Int J Behav Nutr Phys Act. 2011;8:79.

4. The Health and Social Care Information Centre, Health survey for England 2016. 2017.
5. Sport England. Active lives survey 2015-16. UK; 2017. Available from www. sportengland.org/media/11498/active-lives-survey-yr-1-report.pdf. Accessed 10 Aug 2017.

6. Lee PH, Macfarlane DJ, Lam TH, Stewart SM. Validity of the international physical activity questionnaire short form (IPAQ-SF): a systematic review. Int J Behav Nutr Phys Act. 2011:8:115.

7. Troiano RP, Berrigan D, Dodd KW, Masse LC, et al. Physical activity in the United States measured by accelerometer. Med Sci Sports Exerc. 2008;40(1):181-8.

8. The Health and Social Care Information Centre, Health survey for England 2008. 2009.

9. Harris TJ, Owen CG, Victor CR, Adams R, et al. A comparison of questionnaire, accelerometer, and pedometer: measures in older people. Med Sci Sports Exerc. 2009;41(7):1392-402.

10. The IPAQ group. International physical activity questionnaire. Available from: https://sites.google.com/site/theipaq/home. Accessed: 21 Jan 2014.

11. Department of Health, General practice physical activity questionnaire (GPPAQ). 2013

12. NHS. NHS Health Check. [Accessed: 13 Oct 2017]; Available from: http:// www.healthcheck.nhs.uk/.

13. Sardinha LB, Magalhaes JP, Santos DA, Judice PB. Sedentary patterns, physical activity, and cardiorespiratory fitness in association to glycemic control in type 2 diabetes patients. Front Physiol. 2017;8:262.

14. Chudyk AM, McKay HA, Winters M, Sims-Gould J, et al. Neighborhood walkability, physical activity, and walking for transportation: a cross-sectional study of older adults living on low income. BMC Geriatr. 2017;17(1):82.

15. Plasqui $G$, Westerterp KR. Physical activity assessment with accelerometers: an evaluation against doubly labeled water. Obesity (Silver Spring). 2007; 15(10):2371-9.

16. Freedson PS, Melanson E, Sirard J. Calibration of the computer science and applications, Inc. accelerometer. Med Sci Sports Exerc. 1998;30(5):777-81.

17. Fassier P, Zelek L, Partula V, Srour B, et al. Variations of physical activity and sedentary behavior between before and after cancer diagnosis: results from the prospective population-based NutriNet-Sante cohort. Medicine (Baltimore). 2016;95(40):e4629.

18. Barr-Anderson DJ, Flynn Jl, Dowda M, Taverno Ross SE, et al. The modifying effects of race/ethnicity and socioeconomic status on the change in physical activity from elementary to middle school. J Adolesc Health. 2017; 61(5):562-70.

19. Demeyer H, Louvaris Z, Frei A, Rabinovich RA, et al. Physical activity is increased by a 12-week semiautomated telecoaching programme in patients with COPD: a multicentre randomised controlled trial. Thorax. 2017; 72(5):415-23.

20. Ekelund U, Kolle E, Steene-Johannessen J, Dalene KE, et al. Objectively measured sedentary time and physical activity and associations with body weight gain: does body weight determine a decline in moderate and vigorous intensity physical activity? Int J Obes. 2017;41(12):1769-74.

21. Horne D, Kehler DS, Kaoukis $G$, Hiebert B, et al. Impact of physical activity on depression after cardiac surgery. Can J Cardiol. 2013;29(12):1649-56.

22. Nicaise V, Crespo NC, Marshall S. Agreement between the IPAQ and accelerometer for detecting intervention-related changes in physical activity in a sample of Latina women. J Phys Act Health. 2014:11(4):846-52.

23. Baker G, Gray SR, Wright A, Fitzsimons C, et al. The effect of a pedometerbased community walking intervention "walking for wellbeing in the west" on physical activity levels and health outcomes: a 12-week randomized controlled trial. Int J Behav Nutr Phys Act. 2010;7:51.

24. Fuller NR, Williams K, Shrestha R, Ahern AL, et al. Changes in physical activity during a weight loss intervention and follow-up: a randomized controlled trial. Clin Obes. 2014;4(3):127-35.

25. Opdenacker J, Boen F, Vanden Auweele Y, De Bourdeaudhuij I. Effectiveness of a lifestyle physical activity intervention in a women's organization. J Women's Health (Larchmt). 2008;17(3):413-21.

26. Harris T, Kerry SM, Victor CR, Shah SM, et al. PACE-UP (pedometer and consultation evaluation--UP)--a pedometer-based walking intervention with and without practice nurse support in primary care patients aged 45-75 years: study protocol for a randomised controlled trial. Trials. 2013;14:418.

27. Harris T, Kerry SM, Limb ES, Victor CR, et al. Effect of a primary care walking intervention with and without nurse support on physical activity levels in 45- to 75-year-olds: The pedometer and consultation evaluation (PACE-UP) cluster randomised clinical trial. PLoS Med. 2017;14(1):e1002210.

28. The IPAQ group. Guidelines for data processing and analysis of the International Physical Activity Questionnaire (IPAQ) - Short and Long forms. 
[Accessed: 15 Oct 2015]; Available from: https://sites.google.com/site/ theipaq/scoring-protocol.

29. Ahmad S, Harris T, Limb E, Kerry S, et al. Evaluation of reliability and validity of the general practice physical activity questionnaire (GPPAQ) in 60-74 year old primary care patients. BMC Fam Pract. 2015;16:113.

30. StataCorp. Stata Statistical Software: Release 14: StataCorp LLC; 2015. http:// www.stata.com/

31. Department of Health, Start active, stay active: a report on physical activity for health from the four home countries' chief medical officers. 2011, https://www.gov.uk/government/uploads/system/uploads/attachment_ data/file/216370/dh 128210.pdf.

32. World Health Organisation. Global Recommendations on Physical Activity for Health. Geneva: World Health Organisation; 2010. p. 58

33. US Department of Health and Human Services. 2008 Physical activity guidelines for Americans. Washington: Office of Disease Prevention and Health Promotion; 2008. p. 76.

34. Ekelund U, Sepp H, Brage S, Becker W, et al. Criterion-related validity of the last 7-day, short form of the international physical activity questionnaire in Swedish adults. Public Health Nutr. 2006;9(2):258-65.

Ready to submit your research? Choose BMC and benefit from:

- fast, convenient online submission

- thorough peer review by experienced researchers in your field

- rapid publication on acceptance

- support for research data, including large and complex data types

- gold Open Access which fosters wider collaboration and increased citations

- maximum visibility for your research: over $100 \mathrm{M}$ website views per year

At $\mathrm{BMC}$, research is always in progress.

Learn more biomedcentral.com/submissions 